\title{
ACCESS NODE DIMENSIONING FOR IPTV TRAFFIC USING EFFECTIVE BANDWIDTH
}

This article presents a dimensioning of capacity of access node (Black Diamond 12 804R) for IPTV source (6 channels of IPTV), which was one part of our project with Slovak Telecom. We describe IPTV traffic by two mathematic models and use Effective bandwidth for dimensioning of required link capacity with respect to QoS.

Keywords: Markov Modulated Regular Process, M/D/1/queue, Network Transmission Line Dimensioning, Effective Bandwidth, Quality of Service, IPTV Traffic.

\section{Introduction}

With the need of dimensioning the capacity of access node we met while working on a project for Slovak Telecom. It solves the problem of dimensioning link capacity in the network node. The challenge was to dimension the capacity for different scenarios, while we have available four different measurements of IPTV stream outgoing from the source of IPTV service Magio which was acquired in Slovak Telecom laboratory [1]. We had available measurements of monoscope and 1, 6 and 64 channels of IPTV. Our research shows that the recording of 6 channels of IPTV, we can approximate with Poisson distribution, while the coding creates a constant length packets. Proposal for capacity had to take into account the QoS parameters for the analyzed IPTV flow [2]. In agreement with the contracting authority of the research project, we considered only packet lost and delay. This work maps the procedure used in our search for suitable analytical method.

We deal with a model that assumes an infinite length buffer. Random variable $q$ describes the steady-states distribution of the queue. Packet loss indicates the value $P_{\text {lost }}=P(q>n)$, where $n$ is the recommended buffer size, which can be found. The excess value of $\mathrm{X}$ causes packets discard due to unacceptable delay. Input process $A(t)$ is Poisson process with intensity $\lambda, A(t) \sim P o(\lambda t)$, denotes the transmission link capacity $c$. We use a parameter $\rho=$ $=\lambda / \mathrm{c}$, which represents the load or system utilization.

\section{IPTV traffic description}

The basic element of the network is traffic source. There are several concepts how to describe the packet flow generated by this traffic source. If we consider a discrete-time system, the traffic source will be described by arrival process $A($.$) with increments$ $a(t)$, where $A(t)$ is the cumulative number of arrivals by the time $t$ and $a(t)$ is the number of arrivals at time $t$ for the arrival process
$A$ (.) (increments at time $t), a(t)=A(t)-A(t-1)$. Let assume that there is no arrival at time $t-0, A(0)=a(0)=0$ and packets from the traffic source are the same size. When we have the measurement of traffic which is sufficiently long, we can compute important statistical parameters, or create a corresponding stochastic model. Two basic parameters used for traffic description are average rate $\lambda_{\text {avg }}$ and peak rate $\lambda_{\text {peak }}$. It is easy to compute these parameters from the measured traffic with length

$$
\lambda_{\text {avg }}=\rho=\frac{1}{n} \sum_{i=1}^{n} a(i)=\frac{A(n)}{n}, \lambda_{\text {peak }}=\max _{0 \leq t \leq n}[a(t)]
$$

We want to design a stochastic model for the arrival process description. We considered a time slot of $20 \mathrm{~ms}$. The record with increments of packet stream with 6 channels of IPTV service Magio $2000 \mathrm{~ms}$ long is shown in Fig. 1. The entire record we worked with is 2 minutes long.

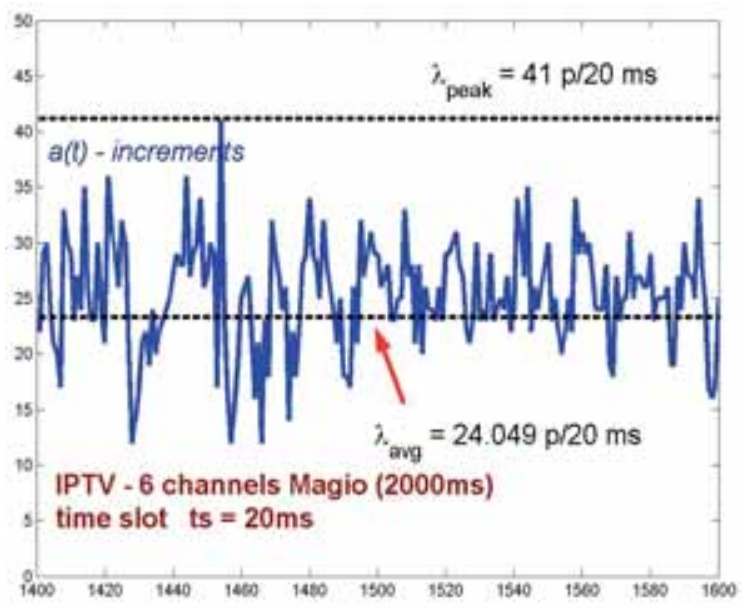

Fig. 16 channels of IPTV service Magio, time slot 20 ms, duration $2000 \mathrm{~ms}$

\footnotetext{
* Juraj Smiesko, Jana Uramova

Department of Information Networks, Faculty of Management and Informatics, University of Zilina, Slovakia, E-mail: juraj.smiesko@fri.uniza.sk
} 
Our task is to find a suitable capacity $c$ for the IPTV stream while we have to meet Quality of service, packet lost $P_{\text {lost }}$ and maximum delay $d$. The value of capacity will be between the mean intensity and the peak value:

$$
\lambda_{\text {avg }} \leq c \leq \lambda_{\text {peak }}
$$

First we find a suitable stochastic model for the measured flow then we will deal with analytical methods for determining the capacity.

For the time slots, we calculated the empirical probability distribution of increments $a(t)$ and we compared their values with Bernoulli and Poisson models. In our research we did experiments with different lengths of time slots. We found that for the size of time slot less than $10 \mathrm{~ms}$ Bernoulli model is very close to the measured data, in all other cases, however, better approximation is achieved by Poisson model. Figure 2 illustrates the already mentioned case with a length of time slot of $20 \mathrm{~ms}$.

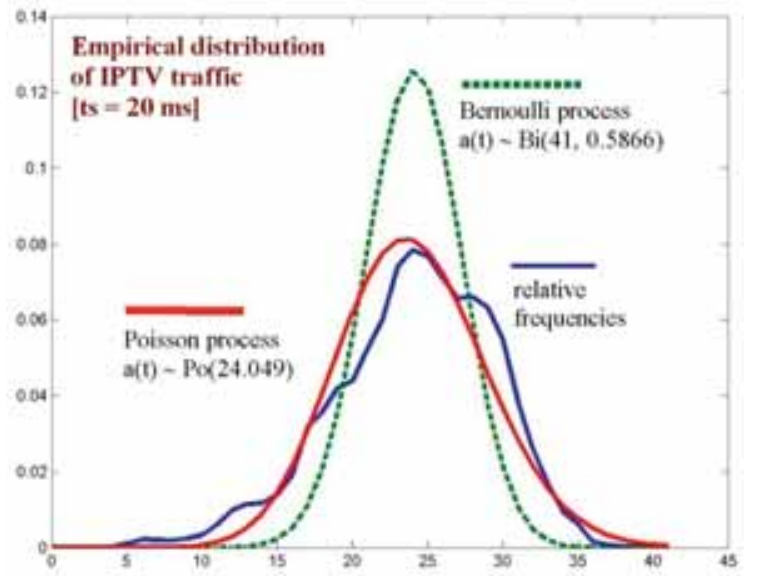

Fig. 2 Two stochastic model for IPTV traffic

For further analysis we decided to use a Poisson model for several reasons. In addition to the above distribution we took into account the fact that the Bernoulli model versus Poisson model provides an upper estimate for the probability of higher values in the measured flow. Our task was to allocate the capacity of the flow with respect to QoS, so we decided for a model with higher probability of peak traffic. Another crucial fact for the choice of the Poisson model was that we wanted to be able to use $\mathrm{M} / \mathrm{D} / 1$ queueing model for the capacity dimensioning.

\section{Queueing system $M / D / 1 /$ queue}

Queueing system $\mathrm{M} / \mathrm{D} / 1$ /queue is well known in Queueing Theory This system has a single queue, Poisson input process (M), constant service distribution (D). These parameters correspond to Poisson model of IPTV flow and constant packet length.

\section{Let probabilities}

$\pi_{k}=P(q=k)$ represent a steady-state distribution of a stabilized system. Parameter $\rho=\lambda / c$ denotes a system load. For the stabilized system $\rho<1$ must be satisfied. Probabilities are derived recurrent equations, [3]:

$$
\begin{aligned}
& \pi_{0}=e^{-\rho}\left[\pi_{0}+\pi_{0}\right], k=1,2,3, \ldots, \\
& \pi_{k}=e^{-\rho}\left[\left[\pi_{0}+\pi_{1}\right] \frac{\rho^{k}}{k !}+\sum_{i=1}^{k} \pi_{i+1} \frac{\rho^{k-i}}{(k-i) !}\right]
\end{aligned}
$$

The formula for probability of packet loss $P_{\text {lost }}=P(q>n)$ for value $n=3,4, \ldots$ is:

$$
\begin{aligned}
& p_{\text {lost }}=1-\left[e^{2 \rho}-\rho e^{\rho}\right] \pi_{0}- \\
& -\sum_{k=3}^{n}\left[e^{\rho} \pi_{k-1}-\frac{\rho^{k-1}}{(k-1) !} e^{\rho} \pi_{0}-\sum_{i=1}^{k-2} \pi_{i+1} \frac{\rho^{k-1-i}}{(k-1-i) !}\right]
\end{aligned}
$$

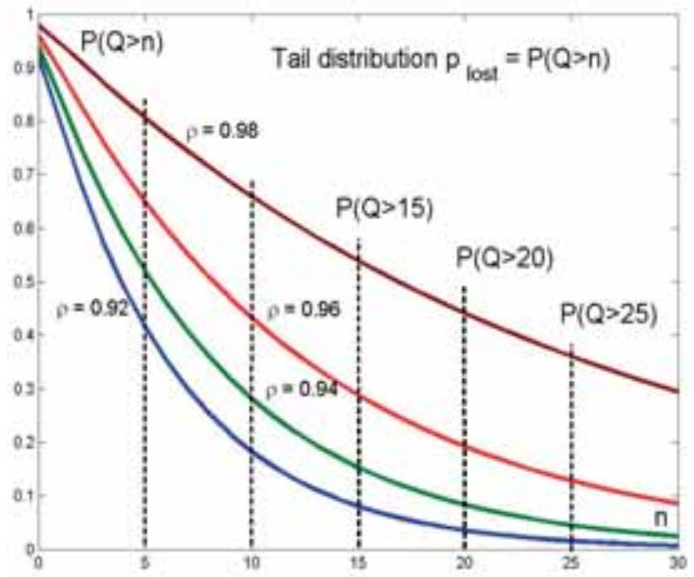

Fig. 3 Probability distribution and tail distribution of different values $\rho$

For the given values of $\rho$ and $P_{\text {lost }}$ we know how to design a maximum accepting buffer size $n$ and consequently a maximum delay using probability queue distribution of model $\mathrm{M} / \mathrm{D} / 1$. See Fig. 3 for illustration.

For example, let $\rho=0.95$ and $P_{\text {lost }}=0.001$. From the expression (1) we gain the maximum accepting queue size $n=47$. Strictly speaking 47 is the first value when the tail distribution decreases under $0.0010 .000961<0.001$. It's easy to compute the needed link capacity and maximum delay for the given average rate $\lambda=$ $=1201.7(p / s): c=\lambda / \rho \cong 1271 \mathrm{p} / \mathrm{s}, d=\mathrm{n} / \mathrm{c}=37 \mathrm{~ms}$.

Our problem is the opposite. For the given average rate, lost probability and maximum delay (or acceptable buffer size) we have to compute a link capacity. But this means that the load of the link $\rho$ is unknown and the calculation according to equation (1) is nec- 
essary to use complicated iterative algorithms. For this reason we decided to use the Theory of Large Deviation Principles and Effective Bandwidth for a traffic flow description.

\section{Effective Bandwidth}

The concept of effective bandwidth (EB) has gained much attention due to the looming gain for network analysis and design. The effective bandwidth of a general cumulative arrival process has been defined [4] as

$$
\begin{aligned}
& \alpha(\theta, t)=\frac{1}{\theta t} \sup _{s \geq 0} E\left[e^{\theta(A(s+t)-A(s))}\right] \\
& 0<\theta, t<\infty
\end{aligned}
$$

depending upon the space parameter $\theta$ and the time parameter $t$ The effective bandwidth provides a convenient tool for analysis and description of data flows. Theory of Large Deviation Principles provides the tool for link dimension with respect to probability of packet loss (see [5])

$$
\begin{aligned}
& \alpha(\theta, t)=c \Leftrightarrow P(q>n) \asymp e^{\theta n} \text { or } \\
& \lim _{n \rightarrow \infty} \frac{\ln P(q>r)}{n}=-\theta
\end{aligned}
$$

where constant $n$ is size of queue (buffer) and variable $q$ is steadystate length of queue. The relation (3) means that if we design a capacity of link equal to the effective bandwidth, than probability of buffer overflow decays exponentially with constant $\theta$.

If the arrival process $\mathrm{A}(t)$ has a stationary increments a $(i)$, we gain a simple form of the effective bandwidth:

$$
\alpha(\theta, t)=\frac{1}{\theta t} \sup _{s \geq 0} \ln E\left[e^{\theta(A(s+t)-A(s))}\right]=\frac{1}{\theta t} \ln E\left[e^{\theta A(t)}\right]
$$

The effective bandwidth has very interesting properties. For example, the average rate, asymptotic variance, peak rate and burst period are included in its Taylor expansion [5]. Further, the value of $\mathrm{EB}$ in $\theta=\mathbf{0}$ equals to the average rate:

$$
\alpha(0, t)=\frac{E[A(t)]}{t}=\lambda_{\text {avg }}
$$

If the process $\mathrm{A}(t)$ has a stationary and above bounded increments, $\forall t, a(t) \leq \lambda_{\text {peak }}$, it is easy to show (using Jensen inequality [6]), that the effective bandwidth is between average rate and peak rate:

$$
\lambda_{\text {avg }} \leq \alpha(\theta, t) \leq \lambda_{\text {peak }}
$$

Let process $\mathrm{A}(t)$ have stationary (identical) and independent increments (i.i.d ) $\mathrm{a}(t)$. This means that the random variables $\mathrm{a}(t)$ have the same probability distribution and are mutually indepen- dent. Let $\varphi_{a}(\theta)=E\left[e^{\theta \alpha(t)}\right]$ be the moment generation function and $\lambda_{a}(\theta)=\ln \square \varphi_{a}(\theta)$ be the cumulative generation function of increments. The effective bandwidth has form:

$$
\alpha(\theta, t)=\frac{1}{\theta t} \ln E\left[e^{\theta \sum_{t=1}^{t} x}\right]=\frac{1}{\theta t} \ln \varphi_{a}(\theta)^{t}=\frac{\lambda_{a}(\theta)}{\theta}
$$

The effective bandwidth of arrival process with i.i.d increments doesn't depend on time parameter and equals to scale cumulative generation function. Then we assign the effective bandwidth as $\alpha(\theta)$. The formula for link dimensioning is

$$
\alpha(\theta)=\frac{\lambda_{a}(\theta)}{\theta}=c \Leftrightarrow P(q>n) \asymp e^{-\theta n}
$$

In many cases of buffer dimensioning for real scenarios, the buffer size $n$ is unknown but allowed delay for traffic is given. When we assume FIFO service policy [7] and put $c \theta=\lambda(\theta)$ we can redesign dimension formula (8) as follows:

$$
d=\frac{n}{c} \Rightarrow P_{\text {lost }}(q>n)=e^{-\theta n}=e^{-\theta d c}=e^{-d \lambda_{a}(\theta)}
$$

We replaced asymptotic decay “ $\asymp$ " by equality “=”. It means we will obtain upper estimation of a queueing tail distribution. Cumulative generation function is strictly convex. This guarantees the existence of inverse function $\lambda^{-1}($.). We obtain the dependence between space parameter $\theta$ and QoS parameters:

$$
\theta_{0}=\lambda^{-1}\left(\frac{\ln p_{\text {lost }}}{-d}\right)
$$

Now we can design a link capacity using $\theta_{0}: c=\frac{\lambda\left(\theta_{0}\right)}{\theta_{0}}$ and we can guarantee upper estimate for values of maximum delay and packet loss probability. Equation (9) we call Formula for Link Dimensioning using Effective Bandwidth with respect to QoS.

\section{Dimensioning capacity for IPTV traffic}

\subsection{Poisson model}

The main basic model for IP traffic is Poisson process. This is process with i.i.d. increments with Poisson distribution. The effective bandwidth for Poisson process is:

$$
\alpha(\theta)=\lambda \frac{e^{\theta}-1}{\theta}
$$

For comparison with steady-state analysis of model M/D/1 we use same inputs parameters, $\rho=0.95$ and $p_{\text {lost }}=0.001$. Now we have to find a numeric solution for equation

$$
\frac{1}{\rho}=\frac{y^{\theta}-1}{\theta} \Rightarrow \theta=0.1016
$$


We compute an acceptable queue length and maximum delay:

$$
\begin{aligned}
& p_{\text {lost }}=e^{-n \theta} \Rightarrow n=\frac{\ln p_{\text {lost }}}{-\theta} \doteq 68 \Rightarrow \\
& d=\frac{n}{c}=\frac{68}{1265}=54 \mathrm{~ms}
\end{aligned}
$$

Using Effective Bandwidth we derive the maximum delay $54 \mathrm{~ms}$ which is more than we have gained from the model using equation (1), $37 \mathrm{~ms}$. On the other hand we can say that for a given setting we guarantee that the maximum delay no more than $54 \mathrm{~ms}$.

Our goal was the opposite, we should determine the required link capacity for the QoS parameters. First, we can compute parameter $\theta$ for a given delay and packet loss using formula (9):

$$
\theta_{0}=\ln \left[d \lambda-\ln p_{\text {lost }}\right]-\ln [d \lambda]
$$

Using the equation (8) we derive the explicit formula for link capacity:

$$
c=F\left(d \cdot p_{\text {lost }}\right)=\frac{\ln p_{\text {lost }}}{d\left[\ln (d \lambda)-\ln \left[d \lambda-\ln p_{\text {lost }}\right]\right]}
$$

Using the average rate of the traffic $\lambda=1201.7(p / s)$ and specified QoS parameters $d=100 \mathrm{~ms}$ and $p_{\text {lost }}=0.00001$ we get:

$$
\theta_{0}=0.091, c=1264.2 \frac{p}{s}, n=d \cdot c \doteq 127, \rho=0.955
$$

We get upper estimate of the required link capacity $1264.2 \mathrm{p} / \mathrm{s}$, or load $\rho=0.955$. We compare the result with the values obtained directly from the model M/D/1. When the load is 0.955 we observe with expression (1) when its value falls below $p_{\text {lost }}, P\left(q>n_{0}\right) \leq$ $\leq 0.00001$.

We obtained the value of buffer size $n_{0}=126$. See Fig. 4 .

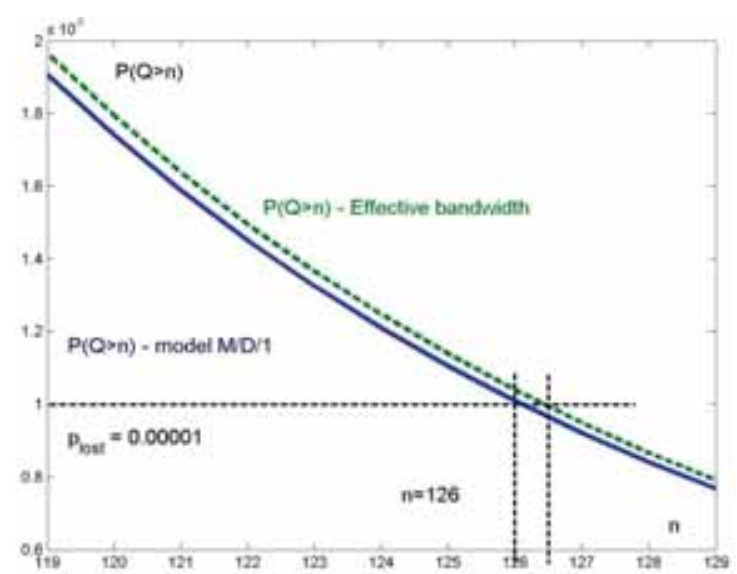

Fig. 4 Tail distribution for $n=119, \ldots, 129$

This is a very pleasing result achieved using the Effective Bandwidth. We get the same result as we obtained by iterative algo- rithms when using the queue distribution of the model M/D/1. Using Effective Bandwidth only as the substitution into the explicit function of capacity was sufficient.

To get the most accurate results for estimating the capacity we will use a more detailed model of the process and describe the IP traffic flow using Markov Modulated Regular Process, MMRP.

\subsection{Markov Modulated Regular Process}

Markov modulated process (MMP) consists of the period On and period Off. If the process is in period On, it produces IP traffic, and if it is in the period Off, it means that nothing is transmitted. The simplest situation is when the process produces a regular (deterministic) flow of ones in period On (Fig. 5). Let's call this process as Markov Modulated Regular Process (MMRP). Whereas the switching between the states On and Off is controlled by Markov chain, MMRP is process with i.i.d. increments $a(i)$, which takes values 0 and 1 ([8]).

First state of the chain describes the period On, second state describes the period Off. Let's designate probabilities of transitions between the states as $p_{1.2}=\alpha$ and $p_{2.1}=\beta$. Let $\pi=\left(\pi_{1}, \pi_{2}\right)$ be a steady-state distribution of Markov chain. Using Queueing theory we compute values of steady-state probabilities ([2]):

Let's create a matrix of the intensities of transitions $Q$ for Markov chain and equations for probability of states for a stabilized chain $\pi$ :

$$
\begin{aligned}
& P=\left(\begin{array}{cc}
1-\alpha & \alpha \\
\beta & 1-\beta
\end{array}\right) \cdot \pi \cdot P=\pi \Rightarrow \\
& \pi_{1}=\frac{\beta}{\alpha+\beta}, \pi_{2}=\frac{\alpha}{\alpha+\beta}
\end{aligned}
$$

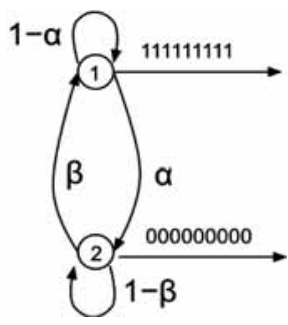

Fig. 5 Markov Modulated Regular Process

Let's calculate a probability distribution of increments in an elementary time slot:

$$
\begin{aligned}
& p_{1}=\pi_{1}(1-\alpha)+\pi_{2} \beta=\pi_{1}, \\
& p_{0}=\pi_{1} \alpha+\pi_{2}(1-\beta)=\pi_{1},
\end{aligned}
$$

Determine the probability distribution of increments in four slots. For slots longer that $m \geq 4$, the situation is even more complicated: 


$$
\begin{aligned}
p_{0} & =\pi_{2}(1-\beta)^{3} \\
p_{1} & =\left(\pi_{1} \alpha+\pi_{2} \beta\right)(1-\beta)^{2}+2 \alpha \beta(1-\beta) \pi_{2} \\
p_{2} & =\left(\pi_{1} \alpha+\pi_{2} \beta\right)(1-\alpha)(1-\beta)+\alpha \beta\left(\pi_{1} \alpha+\pi_{2} \beta+\right. \\
& \left.+\pi_{1}(1-\beta)+\pi_{2}(1-\alpha)\right) \\
p_{3} & =\left(\pi_{1} \alpha+\pi_{2} \beta\right)(1-\alpha)^{2}+2 \alpha \beta(1-\alpha) \pi_{1} \\
p_{4} & =\pi_{1}(1-\alpha)^{3}
\end{aligned}
$$

Our measured IPTV packet flow is sampled to such a maximum time slot so that we obtain no more than 4 packets in one slot. The searched size of time slot is $0.5 \mathrm{~ms}$. The average rate is $\lambda_{\text {avg }}=$ $=0.6(p / 0.5 \mathrm{~ms})$ and, of course, the peak rate is $\lambda_{\text {peak }}=4(p / 0.5 \mathrm{~ms})$. The parameters of MMRP model are gained using Ordinary Least Squares - OLS:

$$
\alpha=0.8065, \beta=0.1423 \Rightarrow \pi_{1}=0.14998, \pi_{2}=0.85002
$$

We compare the obtained empirical probability distribution with the distribution of Poisson process and MMRP. The differential vector size between the empirical distribution and model distribution is used as the error of approximation:

\begin{tabular}{|l|c|c|c|c|c|c|}
\hline Distribution & 0 & 1 & 2 & 3 & 4 & Error \\
\hline Empirical & 0.536 & 0.345 & 0.101 & 0.015 & 0.002 & 0 \\
\hline Poison & 0.548 & 0.329 & 0.099 & 0.020 & 0.003 & 0.020964 \\
\hline MMRP & 0.536 & 0.345 & 0.102 & 0.016 & 0.001 & 0.001370 \\
\hline
\end{tabular}

When using the time slot size of $0.5 \mathrm{~ms}$ we obtained the absolute correlation between the distribution of MMRP model and measured data of 6 channels of IPTV service. We want to show suitable use of MMRP, thus we present a comparison of Effective bandwidths for all three processes. Effective bandwidth for 2-state MMRP has the form ([3]):

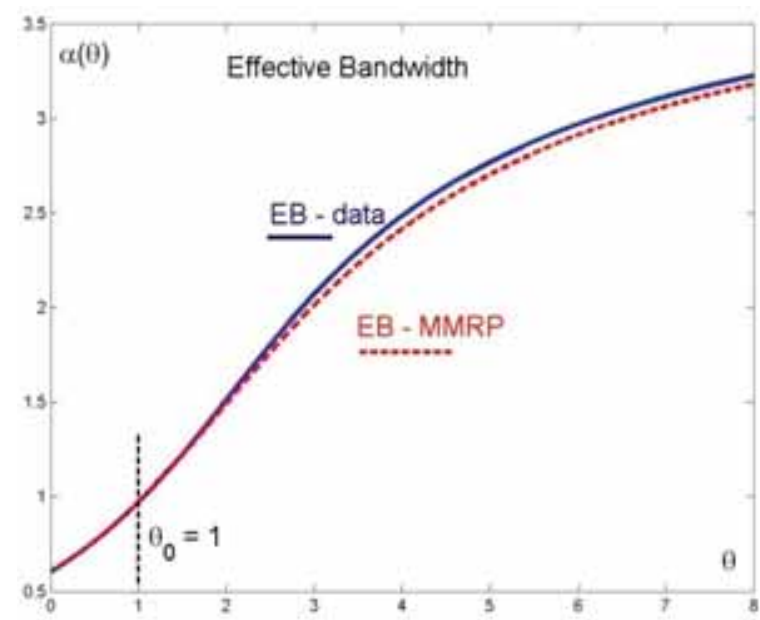

Fig. 6 Effective Bandwidth for MMRP and IPTV traffic

Formula (9) for MRRP model for determining the scale parameter $\theta$ has the form:

$$
\begin{gathered}
\theta_{0}=\ln \left[p_{\text {lost }}^{-1 / d}\right]+\ln \left[p_{\text {lost }}^{-1 / d}-1+\beta\right]- \\
-\ln \left[p_{\text {lost }}^{-1 / d}(1-\alpha)-1+\alpha+\beta\right]
\end{gathered}
$$

For specified parameters $p_{\text {lost }}=0.00001$ a $d=100 \mathrm{~ms}$ we get:

$$
\theta_{0}=0.32819, c=\alpha\left(\theta_{0}\right)=1403.2 \frac{p}{s}, n=d \cdot c \doteq 140,
$$$$
\rho=0.856
$$

The recommended link capacity with respect to specified QoS parameters is substantially higher for MRRP than what we obtained using the Poisson process. However, we can consider this value $c=1403.2(\mathrm{p} / \mathrm{s})$ as more relevant, thanks to the equality of MMRP model with distribution of the measured data of IPTV traffic.

$$
\alpha(\theta)==\frac{1}{\theta} \ln \left[\frac{e^{\theta}(1+\alpha)+(1-\beta)+\sqrt{\left[e^{\theta}(1-\alpha)+(1-\beta)\right]^{2}-4 e^{\theta}(1-\alpha-\beta)}}{2}\right]
$$

Effective bandwidth estimate obtained from the measurements using estimates $\hat{p}_{k}$ :

$$
\alpha(\theta)=\frac{1}{\theta} \ln \left[\sum_{k=0}^{A} e^{\theta k} \hat{p}_{k}\right]
$$

The Effective Bandwidth of MMRP model significantly better approximates the estimate of Effective Bandwidth of measured IPTV traffic (Fig. 6). Although the Effective Bandwidth of MMRP acquires lower values than the real Effective Bandwidth for higher values of scale parameter $\theta$, but in real values of QoS parameters $p_{\text {lost }}$ and $d$ value of the scale parameter $\theta$ does not exceed the value $\theta_{0}=1$ (its our own empirical opinion).

\section{Conclusion}

Our task was to recommend the capacity for access nodes to the core network for measured IPTV traffic flows with respect to QoS parameters. This task was related to research for Slovak Telecom. In this article we analyzed 6 channels of IPTV service for which we came to a Poisson process as an ideal model. But the dimensioning of access node using the classical model $\mathrm{M} / \mathrm{D} / 1$ is proved impractical (frame sizes of IPTV traffic were the same through the use of coding). Therefore, we decided to use the Effective Bandwidth for access node dimensioning. For our purposes we derived the Formula for Link Dimensioning with respect to QoS. To get 


\section{caMMNICOIIONS}

better results we used the Markov Modulated Regular Process (in addition to Poisson process) which reached the absolute conformity with the measured data. The obtained results were tested by simulations and recommended to the contracting authority of the research project as relevant. For the theoretical contribution of our work we consider the derivation of formulas for calculation of a scale parameter using the inverse cumulative function, providing effective bandwidth for the traffic. We found that for specific para- meters typical for IPTV (it means very low probability of packet loss and a relatively high maximum delay) an estimate of the capacity obtained through the Effective bandwidth is very close to the value obtained from the queueing model. In parallel, we analyze the IPTV service with a different number of channels, in addition to 6 channels. The results of this research will be included in subsequent articles.

\section{References}

[1] T-COM: Project: Modeling of IP Traffic, Research report, 2007-2008.

[2] SHIBAB, E., CAI, L.: IPTV distribution technologies in broadband home networks, Proc. IEEE CCECE, Vancouver, BC, Canada, Apr. 2007.

[3] GELENBERE, E., PUJOLLE, G.: Introduction to Queuing Networks, John Wiley \& Sons Ltd., Paris, 1987

[4] KELLY, F. P.: Notes on Effective Bandwidth, Stochastic Networks: Theory and Application, pp. 141-168, Oxford University Press, 1996

[5] SMIESKO, J.: Link Dimensioning with Respect to QoS, J. of Information, Control and Management Systems, Faculty of Management Science and Informatics, University of Zilina, vol. 8, No. 1, 2010

[6] CHANG, CH. SH.: Performance Guarantees in Communication Networks, Springer Verlag, 2000

[7] SZIGETI, T., HATTINGH, CH.: End-to-End QoS Network Design: Quality of Service in LANs, WANs, and VPNs, Cisco Press, 2005.

[8] SMIESKO, J., URAMOVA, J.: IP Traffic Description by Markov Modulated Regular Process, J. of Information, Control and Management Systems, Faculty of Management Science and Informatics, University of Zilina, vol. 1, No. 1, 2010. 